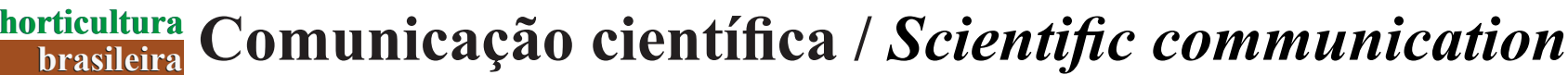

MENEZES JÚNIOR, FOG; KURTZ, C. 2016. Produtividade da cebola fertirrigada sob diferentes doses de nitrogênio e densidades populacionais. Horticultura Brasileira 34: 571-579. DOI - http://dx.doi.org/10.1590/S0102-053620160418

\section{Produtividade da cebola fertirrigada sob diferentes doses de nitrogênio e densidades populacionais}

\author{
Francisco OG Menezes Júnior; Claudinei Kurtz \\ Estação Experimental de Ituporanga (Epagri), Ituporanga-SC, Brasil; franciscomenezes@epagri.sc.gov.br; kurtz@epagri.sc.gov.br
}

\section{RESUMO}

$\mathrm{Na}$ cultura da cebola, diversos trabalhos comprovam que a densidade populacional e a fertilização nitrogenada devem ser consideradas quando se deseja combinar produtividade com produtos de qualidade. $\mathrm{O}$ experimento teve por objetivo avaliar o efeito de doses de nitrogênio, via fertirrigação, em diferentes densidades populacionais na produtividade e armazenamento pós-colheita de bulbos de cebola nas condições do Alto Vale do Itajaí-SC. Com este propósito foram conduzidos dois ensaios de campo, em 2011 e 2013, em Cambissolo Háplico, em Ituporanga-SC. Os tratamentos corresponderam a quatro densidades de plantas $(300,400,500$ e 600 mil plantas/ha) submetidas a cinco doses de nitrogênio $(0,50,100,150$ e $200 \mathrm{~kg} / \mathrm{ha}$ de N), distribuídas ao longo do ciclo vegetativo da cultura via fertirrigação. Utilizou-se a cultivar Bola Precoce. As semeaduras foram realizadas em 25/04/2011 e 19/04/2013, os transplantes em 25/07/2011 e 12/07/2013 e as colheitas em 25/11/2011 e 21/11/2013. Foram avaliadas as produtividades comerciais (total, $\mathrm{Cx} 2, \mathrm{Cx} 3$ e $\mathrm{Cx} 3+$ ), teor de $\mathrm{N}$ na metade do ciclo, florescimento (\%) e perda pós-colheita (\%). Produtividades comerciais totais máximas de 58,3 e 55,1 t/ha são obtidas com doses de 161,3 e 129,0 $\mathrm{kg} /$ ha de N, respectivamente para 2011 e 2013. O aumento de doses de $\mathrm{N}$ reduz a formação de bulbos $\mathrm{Cx} 2$. A maior produtividade $\mathrm{Cx} 3$ (25,6 a 39,3 t/ha) foi obtida com populações de 500 a 600 mil plantas/ ha e doses de 126,1 a $135,9 \mathrm{~kg} / \mathrm{ha}$ de $\mathrm{N}$. O aumento da densidade populacional reduz a produtividade $\mathrm{Cx} 3+$. As maiores produtividades $\mathrm{Cx} 3+(49,6$ a 54,4 t/ha) foram alcançadas com doses de 126,3 e 156,7 $\mathrm{kg} /$ ha de N. Para a cultivar Bola Precoce podem ser considerados adequados na metade do ciclo teores foliares de $\mathrm{N}$ na faixa de 23,0 a $34,0 \mathrm{~g} / \mathrm{kg}$. O aumento das doses de $\mathrm{N}$ concorre para o aumento do florescimento, mas não influencia as perdas em pós-colheita.

Palavras-chave: Allium cepa, plantio direto, nitrogênio foliar, florescimento, conservação pós-colheita.

\section{ABSTRACT}

Onion productivity in fertigated system under different nitrogen levels and population densities

On onion, several studies have confirmed that the population density and nitrogen fertilization should be considered to combine quality and productivity. The experiment aimed to evaluate the effect of nitrogen by fertigation under different population densities on yield and postharvest losses of onion bulbs at Alto Vale do Itajaí, Santa Catarina State, Brazil. Two experiments were carried out, in 2011 and 2013, with Bola Precoce cultivar, over an Haplumbrept soil at Ituporanga, Santa Catarina State. The treatments included four plant densities (300, 400, 500 and 600 plants/ha) and five doses of nitrogen $(0,50,100,150$ and $200 \mathrm{~kg} / \mathrm{ha} \mathrm{N})$, distributed by fertigation. Sowing was carried out on 2011/04/25/ and 2013/04/19, transplanting on $2011 / 07 / 25$ and $2013 / 07 / 12$ and harvest on $2011 / 11 / 25$ and 2013/11/21. Commercial yields (total, Cx2, Cx3 and Cx3+), N content in half the cycle, flowering (\%) and postharvest losses (\%) were evaluated: the highest total commercial yields of 55.1 and $58.3 \mathrm{t} / \mathrm{ha}$ were reached with doses of 129.0 and $161.3 \mathrm{~kg} / \mathrm{ha} \mathrm{N}$, respectively. Increasing doses of $\mathrm{N}$ reduces the formation of $\mathrm{Cx} 2$ bulbs. The highest Cx3 yield (25.6 to $39.3 \mathrm{t} / \mathrm{ha}$ ) was obtained with populations of 500 to 600 thousand plants/ha and doses of 126.1 to $135.9 \mathrm{~kg} / \mathrm{ha} \mathrm{N}$. Increased population density reduces the $\mathrm{Cx} 3+$ productivity. The highest $\mathrm{Cx} 3+$ (49.6 to $54.4 \mathrm{t} / \mathrm{ha}$ ) was achieved with doses of 126.3 and $156.7 \mathrm{~kg} / \mathrm{ha}$ N. To cultivate Bola Precoce can be considered appropriate, in the middle of the cycle, foliar nitrogen content in the range of 23.0 to $34.0 \mathrm{~g} \mathrm{~N} / \mathrm{kg}$. The increase of nitrogen levels contributes to increase blooming, but does not influence the postharvest losses.

Keywords: Allium cepa, no-tillage system, foliar nitrogen, flowering, postharvest.

(Recebido para publicação em 15 de agosto de 2015; aceito em 7 de abril de 2016)

(Received on August 15, 2015; accepted on April 7, 2016)

\begin{abstract}
$\mathrm{A}$ produtividade das espécies oleráceas é fortemente influenciada por fatores ambientais e práticas agronômicas. Na cultura da cebola, diversos trabalhos comprovam que a densidade populacional e a fertilização nitrogenada devem ser consideradas quando se deseja combinar produtividade com produtos de qualidade (Khan et al., 2002; Russo,
\end{abstract}

2008; Abdissa et al., 2011; Kurtz et al., 2013).

Na cebola, o uso de maiores ou menores densidades populacionais interfere em diversos parâmetros qualitativos e quantitativos. Dentre esses, a produtividade, o diâmetro e a massa fresca dos bulbos e sua classificação comercial, o que se refletirá diretamente na rentabilidade do cultivo (Menezes Júnior \& Vieira Neto, 2012). Por sua vez, a disponibilidade de nitrogênio no solo é um fator que também deve ser considerado, por influenciar diretamente a produtividade da cultura (Shojaei et al., 2011; Vilas Boas et al., 2014).

O nitrogênio é um nutriente que está sujeito a várias reações, as quais podem 
reduzir sua disponibilidade na solução do solo e impossibilitar sua absorção pelas plantas. Do nitrogênio aplicado no solo apenas 40 a $50 \%$ são utilizados pelas culturas (Cantarella, 2007). Além do desperdício de fertilizantes, e consequente aumento do custo de produção, o nitrogênio perdido no sistema solo-planta pode resultar em sérios problemas ambientais, contribuindo para a poluição da água e do ar (Marschner, 2012; Abalos et al., 2014).

Dentre as reações a que o nitrogênio está sujeito, a lixiviação é a mais preocupante (Wang et al., 2014), em especial em regiões de alta precipitação pluviométrica, como o extremo sul do Brasil (Kurtz et al., 2013). Por sua vez, a falta de precipitações contribui para a volatilização do $\mathrm{N}$ (adicionado na forma amídica (ureia) em superfície ou amoniacal $\left(\mathrm{NH}_{4}\right)$ em $\mathrm{pH}$ maior que 7,0 ) e menor eficiência da adubação. Devido a isso, o estudo do parcelamento do nutriente tem sido um dos focos de pesquisa.

Comumente, no Brasil, os trabalhos relacionados à aferição do uso de nitrogênio na cebola referem-se a sistemas de cultivo cuja adubação mineral foi fornecida na forma sólida, parcelada em uma ou mais vezes, considerando ou não as variações (demanda do nutriente) existentes entre densidades populacionais (May et al., 2007; Menezes Júnior \& Vieira Neto, 2012; Kurtz et al., 2013). Barbosa Filho et al. (2008), acrescentam que a baixa eficiência de uso de fertilizantes nitrogenados no país se deve à falta de sincronismo entre a época de aplicação e a de maior demanda da planta. Portanto, há necessidade de estudos que possibilitem ajustar a adubação nitrogenada à demanda de nitrogênio, o que significa considerar a dose do nutriente segundo a população de plantas adotada.

Uma das formas de aumentar a eficiência do uso de fertilizantes nitrogenados é seu fornecimento via água de irrigação, a qual permite que as doses sejam ajustadas conforme a necessidade das plantas ao longo do ciclo (Abalos et al., 2014).

Estudos de fertirrigação na cultura da cebola no Brasil ainda são escassos (Vilas Boas et al., 2014). Resende \&
Costa (2009) e Resende et al. (2009) observaram, em Latossolo Vermelho Amarelo Distroférrico para as cultivares Alfa Tropical e Franciscana IPA-10 em densidade populacional aproximada de $667 \mathrm{mil} \mathrm{plantas} / \mathrm{ha}$ e $90 \mathrm{~kg} / \mathrm{ha}$ de $\mathrm{K}_{2} \mathrm{O}$, aumentos lineares na produtividade comercial com o aumento das doses de 0 a $180 \mathrm{~kg} / \mathrm{ha}$ de N. Nessas condições, na dose máxima de $\mathrm{N}$ foram atingidas para a cultivar Alfa Tropical e Franciscana IPA-10 produtividades comerciais de 36,23 e $58,68 \mathrm{t} / \mathrm{ha}$, respectivamente. Gatto (2013), em Latossolo Vermelho Distroférrico, com a cultivar híbrida Bella Vista, em densidade populacional de 500 mil plantas/ha, verificou que as maiores produtividades comerciais foram obtidas com doses de $180 \mathrm{~kg} /$ ha de N.

Neste trabalho, objetivou-se avaliar o efeito de doses de nitrogênio, via fertirrigação, em diferentes densidades populacionais na produtividade e armazenamento pós-colheita de bulbos de cebola nas condições do Alto Vale do Itajaí-SC.

\section{MATERIAL E MÉTODOS}

O trabalho constou de dois experimentos conduzidos em 2011 e 2013 na Epagri/ Estação Experimental de Ituporanga, localizada no município de Ituporanga-

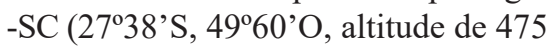
metros). Segundo a classificação de Köeppen, o clima local é do tipo Cfa. Utilizou-se a cultivar Bola Precoce. A cultivar possui como principais características ciclo vegetativo precoce (de 170 a 190 dias), bulbos de cor amarelo-avermethada e forma arredondada, estalo (tombamento natural) em torno de $85 \%$, perda de massa fresca após quatro meses de armazenamento de $24 \%$ e produtividade média de 29,00 a 32,32 t/ha (Gandin et al., 1989, 1994). O solo da área experimental é classificado como Cambissolo Háplico de textura argilosa (Embrapa, 2006). As semeaduras foram realizadas em 25/04/2011 e 19/04/2013, os transplantes em 25/07/2011 e 12/07/2013 e as colheitas em 25/11/2011 e 21/11/2013, respectivamente para os dois experimentos.

As mudas foram produzidas com base nos referenciais tecnológicos propostos pelo Sistema de Produção para a
Cebola (Epagri, 2013). A adubação dos canteiros de produção de mudas constou de $0,5 \mathrm{~kg} / \mathrm{m}^{2}$ de esterco de peru (EP) e $200 \mathrm{~g} / \mathrm{m}^{2}$ da formulação $5-20-10$ e tratamento fitossanitário com os fungicidas pyrimethanil e iprodiona, aplicados nas doses recomendadas pelo fabricante, a cada sete dias, de forma alternada.

As análises químicas do solo das áreas experimentais, realizadas antes da instalação dos ensaios, apresentaram para amostras retiradas na profundidade de 0-20 cm, em 2011 e 2013, respectivamente: argila $=520,0$ e $510,0 \mathrm{~g} / \mathrm{dm}^{3}$; $\mathrm{pH}\left(\mathrm{H}_{2} \mathrm{O}\right)=5,6$ e 6,$6 ; \mathrm{pH}($ índice SMP) $=$ 5,9 e 6,$6 ;$ M.O. $=32,0$ e $33,0 \mathrm{~g} / \mathrm{dm}^{3}$; $\mathrm{P}\left(\right.$ Mehlich-1) $=22,0$ e $10,8 \mathrm{mg} / \mathrm{dm}^{3}$; $\mathrm{H}+\mathrm{Al}=50,0$ e $22,0 \mathrm{mmol} / \mathrm{dm}^{3} ; \mathrm{K}=5,7$ e $3,9 \mathrm{mmolc} / \mathrm{dm}^{3} ; \mathrm{CTC}\left(\mathrm{pH}_{7,0}\right)=164,0 \mathrm{e}$ $126,0 \mathrm{mmol} / \mathrm{dm}^{3} ; \mathrm{Al}=0,5 \mathrm{e} 0,0 \mathrm{mmol} /$ $\mathrm{dm}^{3} ; \mathrm{Ca}=71,0$ e $65,0 \mathrm{mmol} / \mathrm{cm}^{3} ; \mathrm{Mg}=$ 39,0 e $35,0 \mathrm{mmol} / \mathrm{dm}^{3}$.

A análise do solo da área experimental de 2011 indicou a necessidade de calagem. Essa foi realizada no início do mês de abril de 2011 com calcário calcítico ( $40 \%$ de $\mathrm{CaO}, 5 \%$ de $\mathrm{MgO}$ e PRNT $=66,5 \%$ ) na dose de $2,5 \mathrm{t} / \mathrm{ha}$, com o objetivo de elevar o $\mathrm{pH}$ para 6,0 . $\mathrm{O}$ calcário foi distribuído na superfície do solo e incorporado através de aração.

Em abril de cada ano foram semeados nas áreas experimentais o centeio (60 $\mathrm{kg} / \mathrm{ha})$ e o nabo forrageiro $(10 \mathrm{~kg} / \mathrm{ha})$. Dez dias antes do transplante das mudas as plantas de cobertura/adubação verde foram dessecadas com glifosato, sendo as plantas de cobertura/adubação verde deixadas sobre o solo.

Utilizou-se o delineamento experimental de blocos ao acaso, em parcela subdividida, com quatro repetições. Foi alocado nas parcelas o fator adubação nitrogenada e nas subparcelas o fator densidade de plantas. A área total de cada parcela experimental foi de 7,5 $\mathrm{m}^{2}(2,5 \times 3,0 \mathrm{~m})$, tendo como área útil $3,0 \mathrm{~m}^{2}$.

Os tratamentos corresponderam a quatro densidades de plantas $(300,400$, 500 e 600 mil plantas/ha) submetidas a cinco doses de nitrogênio $(0,50,100$, 150 e $200 \mathrm{~kg} / \mathrm{ha}$ de N), distribuídas ao longo do ciclo vegetativo da cultura via fertirrigação.

Os sulcos de transplantio foram abertos com o auxílio de uma sulcadeira 
mecânica. Nestes, tendo por finalidade equiparar os níveis de fósforo no solo, procedeu-se a adubação de plantio com superfosfato triplo na dose de $160 \mathrm{~kg} / \mathrm{ha}$ de $\mathrm{P}_{2} \mathrm{O}_{5}$. Por ocasião do transplante, as mudas foram dispostas em linhas duplas (10 x $10 \mathrm{~cm}$ entre linhas) distanciadas umas das outras em $40 \mathrm{~cm}$. A obtenção da densidade de plantas desejada, populações de 300, 400, 500 e $600 \mathrm{mil}$ plantas/ha, foi realizada reduzindo o espaçamento entre plantas na linha de transplante.

O sistema de fertirrigação, utilizado principalmente para o fornecimento de nutrientes, foi composto por um conjunto motobomba, linha principal condutora (mangueira de $3 / 4$ de polegada) e linha secundária distribuidora (mangueira de $3 / 4$ de polegada) com registros onde foram adaptadas fitas de irrigação com espaçamento entre gotejadores de 10 $\mathrm{cm}$. As fitas gotejadoras foram dispostas no centro das linhas duplas $(10 \times 10 \mathrm{~cm}$ entre linhas) de forma a uniformizar o suprimento de água e nutrientes. A cada fertirrigação semanal foi aplicada uma lâmina de irrigação correspondente a $6,21 \mathrm{~mm}$.

A dose total de nitrogênio, aplicada de acordo com cada tratamento e tendo como fonte o nitrato de amônio, foi parcelada após sete dias do transplante semanalmente conforme a percentagem de absorção do nutriente $(0,9 ; 0,9 ; 2,6$; 4,$1 ; 6,1 ; 8,6 ; 11,1 ; 13,1 ; 13,6 ; 12,5$; 10,$2 ; 7,6 ; 5,3$ e $3,5 \%$ ) tendo por base a curva de absorção da cultivar Crioula Alto Vale determinada por Fayad \& Mondardo (2004), adaptada ao ciclo da cultivar Bola Precoce.

Para todos os tratamentos a adubação potássica foi fornecida via fertirrigação junto à adubação nitrogenada na dose de $90 \mathrm{~kg} / \mathrm{ha}$ de $\mathrm{K}_{2} \mathrm{O}$, sendo utilizado como fonte o cloreto de potássio. $\mathrm{O}$ parcelamento da dose total de potássio seguiu a mesma metodologia descrita para o nitrogênio, ou seja, considerando percentagem de absorção do potássio $(0,6 ; 0,6 ; 1,8 ; 2,9 ; 4,4 ; 6,6 ; 9,0 ; 11,7$; 13,$5 ; 13,9 ; 12,5 ; 10,1 ; 7,4$ e $5,1 \%$ ) com base na curva de absorção da cultivar Crioula Alto Vale adaptada ao ciclo da cultivar Bola Precoce. Aos 60 dias após o transplante (DAT) em 2011, e aos 24 e 60 DAT em duas parcelas em
2013 foram fornecidos via fertirrigação o equivalente a $10 \mathrm{~kg} / \mathrm{ha}$ de sulfato de zinco e ácido bórico. Em 2011, além do zinco e boro, adicionou-se na mesma data e dose mencionadas o sulfato de manganês.

Durante o ciclo da cultura, quando necessário, procedeu-se o controle das plantas indesejáveis com os herbicidas pendimetalina, ioxilina e bentazona nas doses recomendadas pelos fabricantes. Os tratamentos fitossanitários para o manejo de doenças foram realizados em intervalos de 12 a 14 DAT com fungicidas metalaxil-m + mancozebe combinado com ciprodinil ou trifloxistrobina + tebuconazol ou tebuconazol ou iprodiona. Para o manejo de insetos foram realizadas quatro aplicações, a contar do início de outubro, em intervalos de 12 dias, de lambda-cialotrina.

Em 20/10/2011 e 07/10/2013, por ocasião do início da bulbificação (aproximadamente na metade do ciclo), foram coletadas amostras de folhas para avaliação do teor de nitrogênio, amostrando a folha mais nova totalmente expandida de dez plantas por parcela. As folhas foram secas em estufa de ar forçado a $65^{\circ} \mathrm{C}$ até atingir massa constante, sendo a seguir moídas e submetidas à digestão sulfúrica. Nos extratos líquidos foram determinados os teores de $\mathrm{N}$, pelo método de arraste de vapores em aparelho semi-microKjeldahl (Tedesco et al., 1995).

Em 25/11/2011 e 06/12/2013, foram avaliadas as percentagens de florescimento de plantas/bulbos florescidos por área útil $\left(3,0 \mathrm{~m}^{2}\right)$.

A colheita foi realizada quando as plantas apresentavam mais de $70 \%$ de tombamento. Após a cura a campo, feita sete dias após a colheita das plantas, realizou-se a retirada de raízes e o "destalamento" das plantas de forma a deixar uma porção de $1 \mathrm{~cm}$ do pseudocaule. A classificação dos bulbos foi feita com base em seu diâmetro transversal, conforme as normas estabelecidas para a cultura (Brasil, 1995). Para cada classe, mediu-se a massa fresca de bulbos. Determinou-se, então, a produtividade comercial total $(\mathrm{PCT}=\mathrm{Cx} 2+\mathrm{Cx} 3++$ $\mathrm{Cx}>3)$, classe $2(\mathrm{Cx} 2)$, classe $3(\mathrm{Cx} 3)$, classe acima de $3(\mathrm{Cx}>3)$, e classe 3 e superiores $(\mathrm{Cx} 3+=\mathrm{Cx} 3+\mathrm{Cx}>3)$. Para tal foram colhidas plantas de três linhas duplas centrais de cada parcela experimental (parcela útil de 3,0 $\mathrm{m}^{2}$ ). Após a classificação e pesagem, os bulbos foram dispostos em caixas plásticas de armazenamento e levados ao estaleiro onde permaneceram por quatro meses. Findo esse período realizou-se a análise de conservação pós-colheita, a qual considerou percentagem da perda de massa fresca inicial dos bulbos.

Durante os experimentos registraram-se na Estação Meteorológica da Estação Experimental da Epagri de Ituporanga-SC valores médios, em 2011 e 2013 , respectivamente, de: $79,2 \%$ e $79,1 \%$ de umidade relativa do ar; 853,5 e 746,6 $\mathrm{mm}$ de precipitação; $11,4 \mathrm{e}$ $11,0^{\circ} \mathrm{C}$ de temperatura mínima média do ar, 16,5 e $15,8^{\circ} \mathrm{C}$ de temperatura média do ar, e 23,5 e $22,3^{\circ} \mathrm{C}$ de temperatura máxima média do ar (figuras 1A e 1B).

Os dados foram submetidos à análise de variância (teste $\mathrm{F}$ ) e regressão com o uso do programa estatístico SANEST (Zonta \& Machado, 1984). Os dados de percentagem foram transformados em arco-seno raiz de $\mathrm{P} / 100$ para efeitos de análise, sendo apresentadas nos resultados as médias originais.

\section{RESULTADOS E DISCUSSÃO}

\section{Produtividade e seus componentes}

As variáveis de produtividade indicam variações entre os anos agrícolas e seu comportamento (ausência ou presença de interação) em relação às doses de nitrogênio (DN) aplicadas e densidades populacionais (DP) adotadas.

Nos dois anos agrícolas, a análise de variância indicou ausência de interação $(p>0,05)$ entre DN e DP para a produtividade comercial total (PCT). Em 2011 e 2013, o aumento da densidade populacional (DP) não influenciou ( $p>0,05)$ a PCT, cujos rendimentos médios foram de 49,8 e 47,2 t/ha, respectivamente. Menezes Júnior \& Vieira Neto (2012), em sistema convencional com a cultivar Juporanga, ao variarem a DP de 200 a $600 \mathrm{mil}$ plantas/ha para uma mesma dose de $\mathrm{N}$ (75 kg/ha de N), também não observaram variações significativas entre as densidades populacionais para a variável PCT, obtendo produtividade 
média de 36,2 t/ha. May et al. (2007), verificaram o mesmo comportamento para populações de 600.000 a 1.080 .000 plantas/ha e doses de $\mathrm{N}$ de 0 a $150 \mathrm{~kg} /$ ha de $\mathrm{N}$ e atribuíram tal comportamento à compensação entre a massa do bulbo obtida por menores densidades populacionais. Estes autores indicam, entretanto, a necessidade de fertilização nitrogenada diferenciada para cada população com vistas a alcançar a massa de bulbo comercialmente desejável.

Nos dois experimentos, observaram-se relações quadráticas entre as doses de nitrogênio aplicadas e a PCT máxima (Figura 2A). As PCT máximas obtidas em 2011 e 2013, de 58,3 e 55,1 t/ha foram atingidas quando da adição de 161,3 e $129,0 \mathrm{~kg} / \mathrm{ha}$ de $\mathrm{N}$, respectivamente. Isso significa que doses superiores a $161,3 \mathrm{~kg}$ /ha não aumentam a PCT. Kurtz et al. (2013), em sistema de plantio direto e adubação convencional (adubo nitrogenado aplicado no plantio e em cobertura) para a cultivar Bola Precoce, ao adicionar doses crescente de $\mathrm{N}$ (de 0 a $200 \mathrm{~kg} / \mathrm{ha}$ ) observaram o mesmo comportamento quadrático em DP de 250 mil plantas/ha. Nesse caso, os autores obtiveram em duas safras consecutivas, produtividades máximas de 37,9 e 41,6 $\mathrm{t} / \mathrm{ha}$ com doses de 135,5 e $113,0 \mathrm{~kg} / \mathrm{ha}$ de $\mathrm{N}$, respectivamente. May et al. (2007), ao estudar a produtividade da cebola em função da adubação nitrogenada e população de plantas para as cultivares híbridas Optima e Superex, em Latossolo Vermelho Amarelo, estimaram que para a obtenção de 64,8 e 71,0 t/ha devem ser fornecidas quantidades de 125 e $105 \mathrm{~kg} /$ ha de $\mathrm{N}$, respectivamente.

A maior produtividade alcançada no presente experimento, superior na média dos dois anos em $25,4 \%$ ao valor observado por Menezes Júnior \& Vieira Neto (2012) e 31,7\% em relação a Kurtz et al. (2013), e 16,5\% inferior a May et al. (2007) pode ser atribuída às doses, metodologia de adição e parcelamento do $\mathrm{N}$ (distribuição via água de irrigação de doses semanais ao longo do ciclo conforme curva de absorção de nutrientes), bem como diferenças produtivas entre cultivares, além de fatores climáticos relacionados ao ano agrícola ou local de cultivo.

Em relação a sistemas fertirrigados,

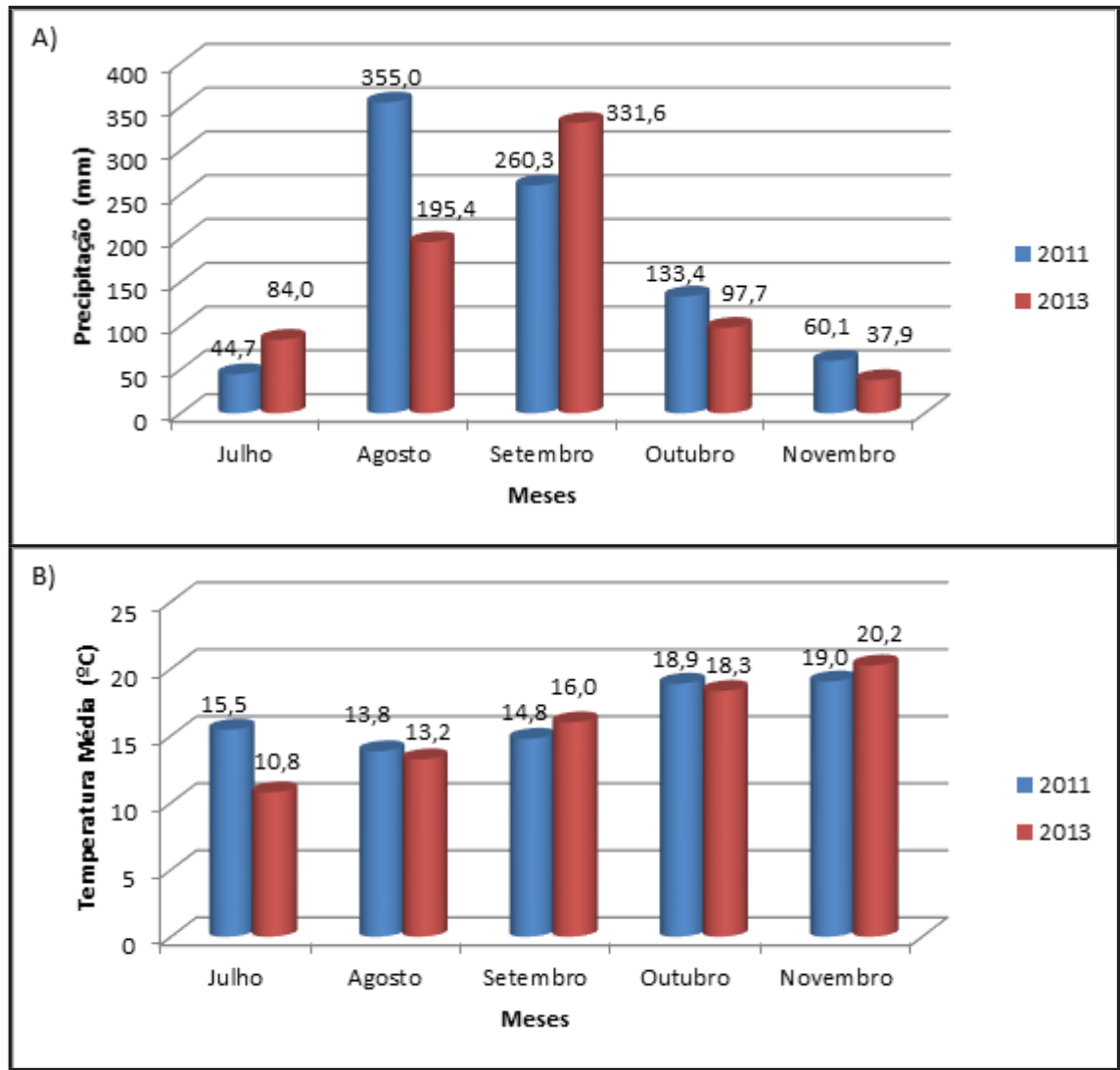

Figura 1. Precipitação mensal acumulada (A) e temperatura media do ar (B), do transplante à colheita, em 2011 e 2013 \{accumulated monthly precipitation (A) and average air temperature (B), from the transplant to harvest, in 2011 and 2013\}. Ituporanga, Epagri, 2013.

Resende \& Costa (2009) e Resende et al. (2009) observaram, em Latossolo Vermelho Amarelo Distroférrico para as cultivares Alfa Tropical e Franciscana IPA-10 em densidade populacional aproximada de $667 \mathrm{mil}$ plantas/ha e $90 \mathrm{~kg} / \mathrm{ha}$ de $\mathrm{K}_{2} \mathrm{O}$, aumentos lineares na produtividade comercial com o aumento das doses de 0 a $180 \mathrm{~kg} / \mathrm{ha}$ de N. Nessas condições, na dose máxima de $\mathrm{N}$, foram atingidas para as cultivares Alfa Tropical e Franciscana IPA-10, produtividades comerciais de 36,23 e $58,68 \mathrm{t} / \mathrm{ha}$, respectivamente. Por sua vez, Gatto (2013), estudando a eficiência de lâminas de irrigação por gotejamento, obteve para cultivar híbrida Bella Vista, em densidade populacional de 500 mil plantas/ ha, produtividade comercial máxima de $70,6 \mathrm{t} / \mathrm{hacom}$ a dose de $180 \mathrm{~kg} / \mathrm{ha}$ de $\mathrm{N}$. No presente estudo, o sistema de fertirrigação foi utilizado basicamente para o fornecimento de nutrientes, o que não garante que a precipitação pluviométrica no período tenha sido suficiente à máxima expressão do potencial produtivo da cultivar Bola Precoce. Contudo, as produtividades máximas obtidas podem ser consideradas elevadas para as condições do Alto Vale do Itajaí e do Brasil, cujas médias nos últimos anos se situam ao redor de $25 \mathrm{t} / \mathrm{ha}$.

Portanto, as variações de produtividades comerciais máximas para DP e doses de nitrogênio empregadas por outros autores (Resende \& Costa, 2009; Resende et al., 2009; Gatto, 2013) estão associadas às condições edafoclimáticas locais, cultivares e manejo da água adotados.

Para a produtividade de bulbos da classe 2 (Cx2), verificou-se interação significativa $(p>0,01)$ entre doses $e$ populações em 2011. Nesse ano, para populações de $300 \mathrm{mil} \mathrm{plantas/ha,} \mathrm{o}$ aumento das doses de $\mathrm{N}$ não interferiu na formação de bulbos $\mathrm{Cx} 2$ (média de 1,59 t/ha), o que está associado à menor DP. Em geral, para as demais DP, houve redução da produtividade de $\mathrm{Cx} 2$ com o aumento das doses de nitrogênio (Figura 2B). As menores produtividades de $\mathrm{Cx} 2$ 


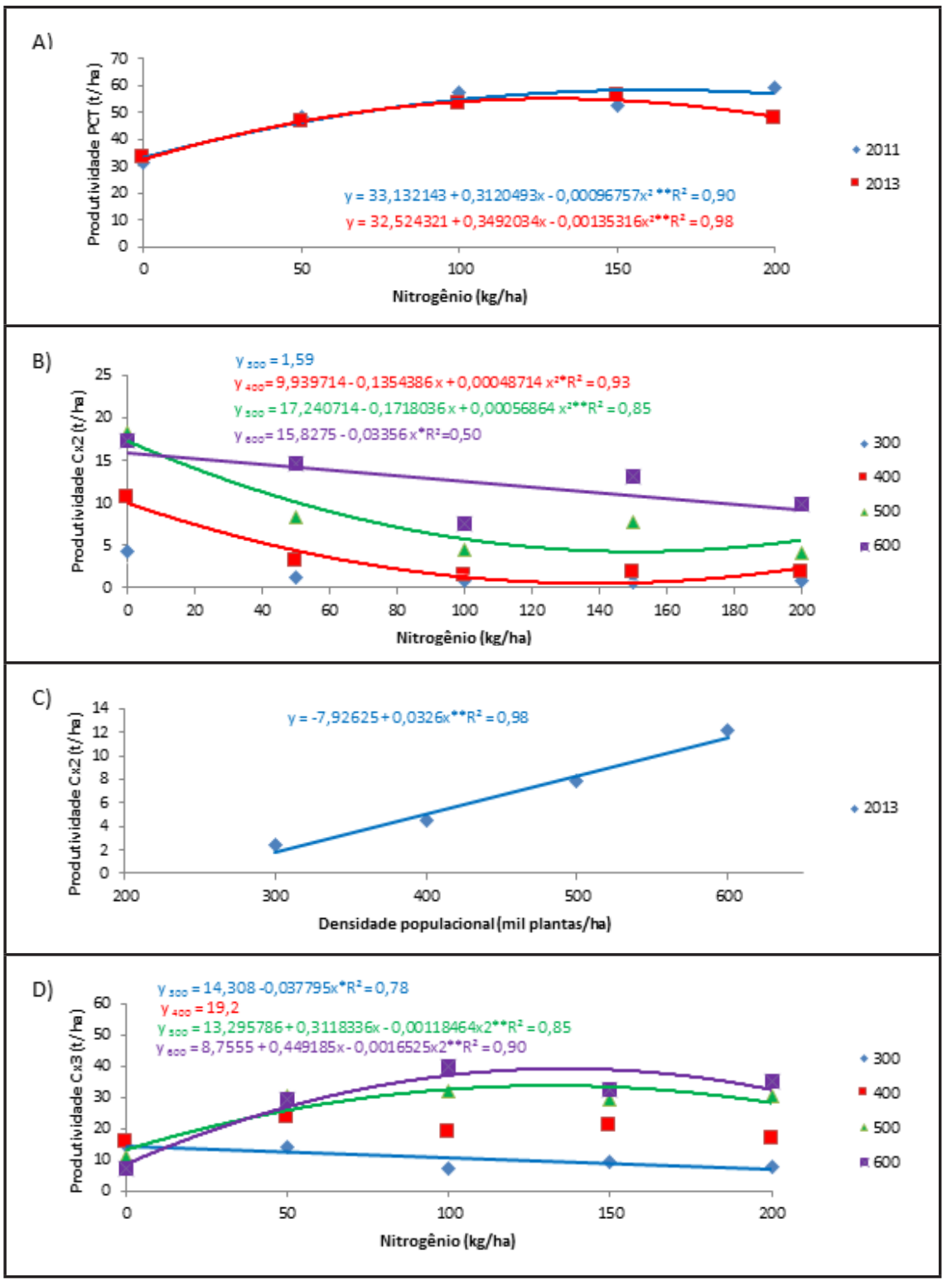

Figura 2. $\mathrm{A}=$ produtividade comercial total $(\mathrm{PCT}) \mathrm{x}$ dose de $\mathrm{N}$ em 2011 e 2013 ; $\mathrm{B}=$ classe $\mathrm{Cx} 2 \mathrm{x}$ dose de $\mathrm{N}$ em 2011; $\mathrm{C}=$ classe $\mathrm{Cx} 2 \mathrm{x}$ densidade populacional em 2013; $\mathrm{D}=$ classe $\mathrm{Cx} 3 \mathrm{x}$ dose de $\mathrm{N}$ em $2011\left({ }^{*} \mathrm{p}>0,05 ;{ }^{*} \mathrm{p}>0,01\right)\{\mathrm{A}=$ total marketable yield (PCT) $\mathrm{N}$ rate in 2011 and 2013; $\mathrm{B}=$ class $\mathrm{Cx} 2 \times \mathrm{N}$ rate in 2011; $\mathrm{C}=$ class $\mathrm{C} \times 2 \times$ population density in 2013; $\mathrm{D}=$ class $\mathrm{Cx} 3 \mathrm{x}$ N rate in $2011(* \mathrm{p}>0,05 ; * * \mathrm{p}>0,01)\}$. Ituporanga, Epagri, 2013.

foram de $0,5(139,0 \mathrm{~kg} / \mathrm{ha}$ de $\mathrm{N}), 4,3$ $(151,1 \mathrm{~kg} / \mathrm{ha} \mathrm{de} \mathrm{N})$ e $9,1 \mathrm{t} / \mathrm{ha}(200,0 \mathrm{~kg} /$ ha de N), para populações de 400,500 e 600 mil plantas/ha, sendo observadas relações quadráticas entre os fatores para as duas primeiras DP e linear para a última. Verifica-se, portanto, que para DP de 400 e 500 mil plantas/ha, doses de $\mathrm{N}$ acima dos níveis mencionados para cada população não foram capazes de reduzir a produtividade $\mathrm{Cx} 2$, enquanto para DP de 600 mil plantas houve tendência da redução até a dose máxima de $\mathrm{N}$ fornecida (Figura 2B).
Em 2013, o aumento das doses de N proporcionou a redução linear de bulbos $\mathrm{Cx} 2$, sendo obtida a menor produtividade $(4,2 \mathrm{t} / \mathrm{ha})$ na dose máxima de $\mathrm{N}$ $\left(\mathrm{y}_{\mathrm{C} x 2+2013}=-9,2445-0,0250413 \mathrm{x}, \mathrm{R}^{2}=\right.$ $0,86)$. Por sua vez, de forma inversa, aumento da DP aumentou a produtividade de bulbos da classe Cx2 (Figura 2C). Esse resultado é concordante com o de outros autores, os quais observaram a mesma tendência ao aumentar a densidade populacional de suas lavouras (Dellacecca \& Lovato, 2000; Shock et al., 2004; Menezes Júnior \& Vieira
Neto, 2012).

A importância da redução da produtividade $\mathrm{Cx} 2$ está relacionada ao preço pago ao produtor, uma vez que, segundo Menezes Júnior \& Vieira Neto (2012), na região do Alto Vale do Itajaí, o produto assim classificado recebe metade da remuneração em relação a bulbos das classes superiores (de diâmetro transversal maior que $50 \mathrm{~mm}$ ). Portanto, em termos mercadológicos para o cebolicultor é importante a redução da produtividade de bulbos $\mathrm{Cx} 2$.

Em 2011 e 2013, a análise de variância revelou interação $(p>0,01)$ entre os fatores analisados para produtividade de bulbos da classe 3 (Cx3). No primeiro ano, estas foram observadas para populações de 300, 500 e 600 mil plantas/ ha (Figura 2D). Para população de 400 mil plantas/ha, independente das doses de $\mathrm{N}$ aplicadas não houve variações de produtividade $\mathrm{Cx} 3$, cuja média foi de 19,2 t/ha. Por sua vez, o aumento das doses de $\mathrm{N}$ reduziu linearmente a produtividade $\mathrm{Cx} 3 \mathrm{em}$ DP de $300 \mathrm{mil}$ plantas/ha, sendo máxima na dose de 0 $\mathrm{kg} / \mathrm{ha}$ de $\mathrm{N}(14,3 \mathrm{t} / \mathrm{ha})$.

Bulbos da classe $\mathrm{Cx} 3$ são aqueles considerados pelo mercado nacional de maior qualidade, evidenciando que a adição de $\mathrm{N}$ em DP de 300 mil plantas/ ha prejudicou a obtenção de bulbos Cx3 e proporcionou a formação de bulbos pertencentes a classes de maior diâmetro. Em populações de 500 e 600 mil plantas/ha, observaram-se relações quadráticas com a adição de $\mathrm{N}$, sendo as produtividades mais elevadas obtidas com doses de 131,6 (33,8 t/ha) e 135,9 $\mathrm{kg} / \mathrm{ha}$ de $\mathrm{N}(39,3 \mathrm{t} / \mathrm{ha})$, respectivamente, indicando que doses superiores a esses limites reduzem a formação de bulbos Cx3.

Em 2013, não foram verificadas influencias da dose de N para DP de 300, 400 e 500 mil plantas/ha, cujas produtividades médias $\mathrm{Cx} 3$ estabeleceram-se em 8,4, 14,7 e 19,1 t/ha. Contudo, relações quadráticas foram observadas para populações de $600 \mathrm{mil}$ plantas/ha com a máxima produtividade de bulbos Cx3 (25,6 t/ha) observada na aplicação de $126,1 \mathrm{~kg} /$ ha de $\mathrm{N}$ (Figura 3A).

Deste modo, considerando os dois anos agrícolas, observa-se que a maior 


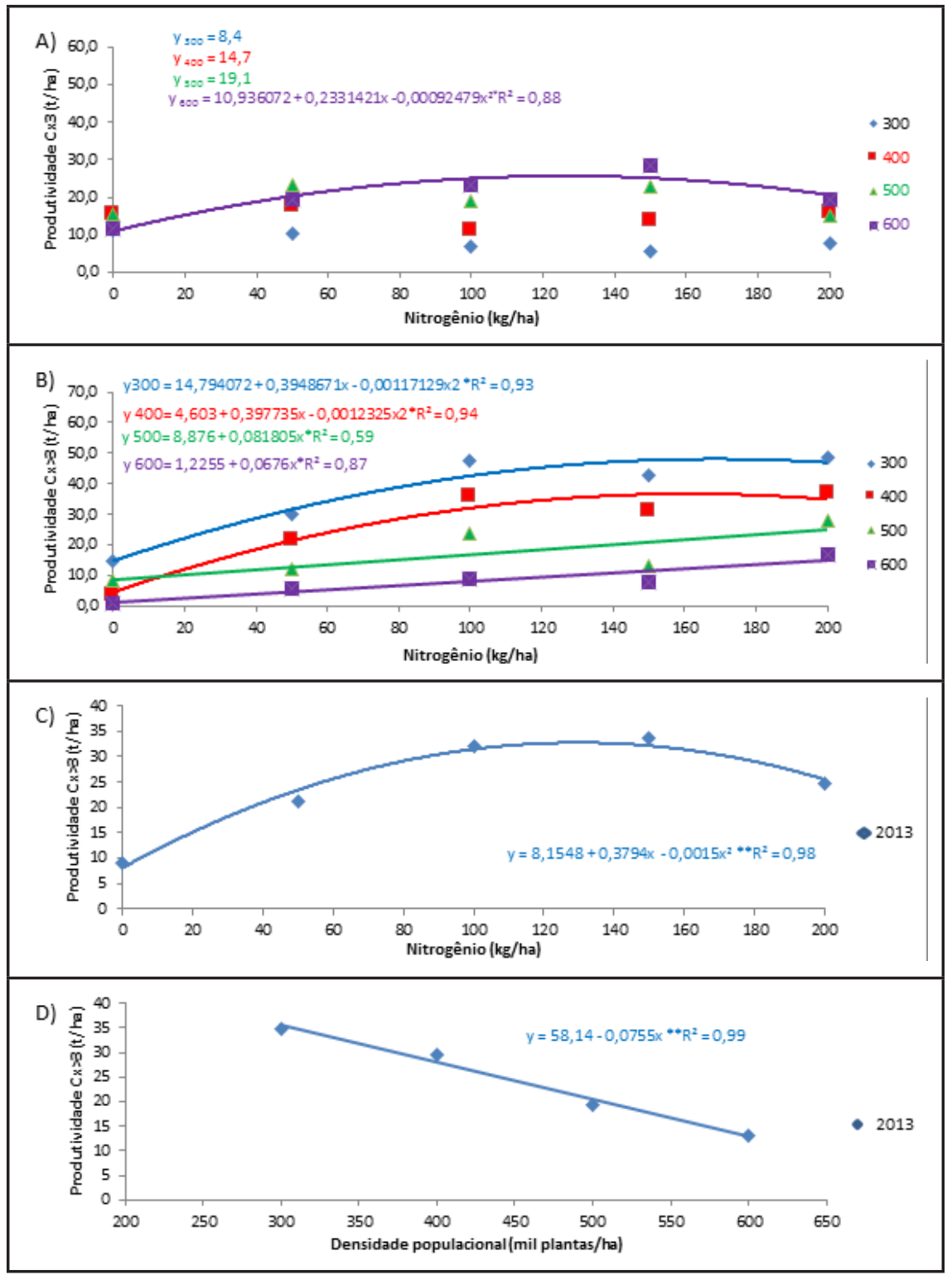

Figura 3. $A=$ produtividade classe $\mathrm{Cx} 3 \mathrm{x}$ dose de $\mathrm{N}$ em 2013; $\mathrm{B}=$ classe $\mathrm{Cx}>3 \mathrm{x}$ dose de $\mathrm{N}$ em 2011; $C=$ classe $C x>3 x$ dose de $N$ em 2013; $D=$ classe $C x>3 x$ densidade populacional em $2013\left({ }^{*} \mathrm{p}>0,05 ; * * \mathrm{p}>0,01\right)\{\mathrm{A}=$ yield class $\mathrm{Cx} 3 \times \mathrm{N}$ rate in $2013 ; \mathrm{B}=$ class $\mathrm{Cx}>3 \times \mathrm{N}$ rate in 2011; $\mathrm{C}=$ yield class $\mathrm{Cx}>3 \mathrm{x} \mathrm{N}$ rate in 2013; $\mathrm{D}=$ class $\mathrm{Cx}>3 \mathrm{x}$ population density in $2013(* \mathrm{p}>0,05 ; * * \mathrm{p}>0,01)\}$. Ituporanga, Epagri, 2013.

produtividade de bulbos $\mathrm{Cx} 3$ (variável de 25,6 a $39,3 \mathrm{t} / \mathrm{ha}$ ) foi obtida com populações de 500 a 600 mil plantas/ ha, com doses de nitrogênio de 126,1 a $135,9 \mathrm{~kg} / \mathrm{ha}$.

Em 2011 foi observada interação $(p>0,01)$ entre doses de $\mathrm{N}$ e DP para a produtividade de bulbos de maior diâmetro transversal $(\mathrm{Cx}>3)$. Para populações de 300 e $400 \mathrm{mil}$ plantas/ha, as relações quadráticas indicaram que as produtividades máximas de 48,1 e 36,7 $\mathrm{t} /$ ha foram obtidas com doses de 168,6 e $161,4 \mathrm{~kg} / \mathrm{ha}$ de N, respectivamente. Por sua vez, em populações de 500 e 600 mil plantas/ha, as relações lineares mostraram produtividades máximas de $25,2 \mathrm{e}$ $14,7 \mathrm{t} /$ ha na maior dose de $\mathrm{N}$ aplicada (200 kg/ha de N) (Figura 3B). Em 2013, a ausência de interação entre os fatores mostrou máxima produtividade $\mathrm{Cx}>3$ (32,8 t/ha) obtida com a dose de $\mathrm{N}$ de $129,7 \mathrm{~kg} / \mathrm{ha}$ de N(Figura 3C), enquanto em relação às densidades populacionais, a maior $(35,5 \mathrm{t} / \mathrm{ha})$ e menor $(12,8 \mathrm{t} / \mathrm{ha})$ produtividade $\mathrm{Cx}>3$ foram obtidas em DP de 300 e 600 mil plantas/ha, respectivamente (Figura 3D).
Portanto, de forma geral, o aumento da DP reduz a produtividade $\mathrm{C}>3$ e doses de $\mathrm{N}$ a partir de $129,7 \mathrm{~kg} / \mathrm{ha}$ de $\mathrm{N}$ tendem a aumentá-la. Observa-se que em menores DP a adição de $\mathrm{N}$ concorre para deslocar a classificação de bulbos para as classes superiores $(C>3)$ em detrimento das demais.

Nos dois anos agrícolas, a análise de variância indicou ausência de interação entre doses de nitrogênio e densidades populacionais (DP) para a produtividade $\mathrm{C} \times 3+$, ou seja, soma da produtividade de bulbos com diâmetro transversal acima de $50 \mathrm{~mm}$. É importante mencionar que na região do Alto Vale do Itajaí, bulbos $\mathrm{Cx} 3+$ são aqueles que recebem no mercado a maior remuneração em nível de agricultor.

Para a produtividade $\mathrm{Cx} 3+$, observaram-se relações quadráticas entre as doses de nitrogênio aplicadas (Figura 4A). As Cx3+ máximas obtidas em 2011 e 2013, de 54,4 e 49,6 t/ha foram atingidas quando da adição de 156,7 e 126,3 kg/ ha de $\mathrm{N}$, respectivamente. Isso significa que doses superiores a $156,7 \mathrm{~kg} / \mathrm{ha}$ não aumentam a $\mathrm{Cx} 3+\mathrm{e}$, da mesma forma que para a PCT, possivelmente, estabeleça um limite de resposta das plantas em produtividade à dose do nutriente em sistema fertirrigado. Por sua vez, o aumento da densidade populacional (DP) influenciou $(\mathrm{p}>0,01)$ a produtividade $\mathrm{Cx} 3+$, a qual foi reduzida linearmente com o aumento da DP (Figura 4B). Portanto, as maiores produtividades $\mathrm{Cx} 3+$ foram obtidas em menores DP.

A maior remuneração recebida pelo produtor pode ser considerada uma vantagem no mercado de Santa Catarina. Contudo, para produtos considerados de melhor qualidade em nível nacional o uso de DP baixas conduzirá a um aumento excessivo de bulbos $\mathrm{Cx} 3+$ em detrimento da formação de bulbos $\mathrm{Cx} 3$ (Figuras 3D e 4B).

Assim, de acordo com os resultados obtidos, observa-se a semelhança de outros autores que, quanto maior a população de plantas, maior será a produtividade Cx2 (Stoffella, 1996; Dellacecca \& Lovato, 2000; Khan et al., 2002; Brewster, 2008; Menezes Júnior \& Vieira Neto, 2012), e maiores quantidades de nitrogênio serão necessárias para a redução de bulbos $\mathrm{Cx} 2$ e manutenção 
ou aumento da produtividade de bulbos $\mathrm{Cx} 3, \mathrm{Cx}>3$ e $\mathrm{Cx} 3+$. (Khan et al., 2002; May et al., 2007; Abdissa et al., 2011).

As variações de produtividade obtidas em 2011 e 2013 indicam que além da população e dose de nitrogênio, outros fatores, como a disponibilidade de água (precipitação) e condições meteorológicas, tenham influenciado a produtividade, uma vez que existem limites na combinação dos fatores em estudo (DP e DN) à obtenção de bulbos considerados de melhor qualidade comercial (Cx3). Resultados semelhantes de pesquisa têm sido obtidos por outros autores (Mansouri et al., 2014).

Em 2011, ano em que foram obtidas as maiores produtividades comerciais totais (média de 49,8 t/ha e máxima de 58,3 t/ha), observaram-se temperaturas do ar (mínima, média e máxima) e precipitação superiores às registradas em 2013 (Figuras 1A e 1B). Supõe-se, portanto, que o aumento do metabolismo vegetal, devido à temperatura, somado à maior disponibilidade de $\mathrm{N}$ (maior disponibilidade de água), tenham contribuído a maiores PCT em 2011.

\section{Teor de $\mathbf{N}$ foliar (NF)}

Para os teores de nitrogênio foliar (NF) não foram observadas interações significativas $(p>0,05)$ entre os fatores (DN e DP) e diferenças significativas entre as densidades populacionais (DP). Em 2011 e 2013, os teores médios de $\mathrm{NF}$ nas DP foram de 23,4 e 27,1 g/kg, respectivamente. Esses níveis situam-se na faixa de nitrogênio considerada adequada para a cultura da cebola, que varia de 19 a $40 \mathrm{~g} / \mathrm{kg}$ (Jones Júnior et al., 1991; Caldwell et al., 1994; Malavolta et al., 1997).

Nos dois anos agrícolas, o aumento das doses de nitrogênio aplicadas ao solo aumentou de forma linear os teores de $\mathrm{N}$ nas folhas $\left(\mathrm{y}_{\mathrm{NF}, 2011}=19,58625+\right.$ $0,03805 \mathrm{x}, \mathrm{R}^{2}=0,97 ; \mathrm{y}_{\mathrm{NF}, 2013}=22,64+$ $\left.0,044375 x, R^{2}=0,92\right)(p>0,01)$. Kurtz et al. (2013), ao aumentar em sistema plantio direto os níveis de nitrogênio (de 0 a $200 \mathrm{~kg} / \mathrm{ha}$ ) em populações de $250 \mathrm{mil}$ plantas/ha observaram para a cultivar Bola Precoce teores foliares (três anos de cultivo) de 23,0 a 29,9 g/ $\mathrm{kg}$. Por sua vez, Gonçalves et al. (2014) verificaram para a mesma cultivar, em populações de 200 mil plantas, teores foliares médios (dois anos de cultivo) de $\mathrm{N}$ de $33,9 \mathrm{~g} / \mathrm{kg}$. Considerando estes aspectos, NF e produtividades obtidas no presente ensaio, teores de $\mathrm{N}$ na faixa de 23,0 a 34,0 g/ $\mathrm{kg}$, podem ser considerados adequados na metade do ciclo da cultura (início da fase bulbificação) para a cultivar em estudo.

\section{Florescimento (FLOR)}

Em 2011, os dados da análise de variância não indicaram interações significativas $(p>0,05)$ entre doses de $\mathrm{N}$ e população em relação à percentagem de florescimento. Observou-se, no entanto, que o aumento das doses de $\mathrm{N}$ concorreu para o aumento linear do florescimento (Figura 4C). Em 2013, houve interação significativa $(\mathrm{p}>0,05)$ entre os fatores, não existindo variações para a DP de $300 \mathrm{mil}$ plantas/ha, cuja média de florescimento foi de $5,6 \%$. Para as demais DP, à semelhança de 2011, verificou-se que o aumento da dose de $\mathrm{N}$ aumentou de forma linear a floração, quanto maior a densidade populacional (Figura 4D).

Os resultados obtidos em 2011 e 2013 são discordantes dos de Kurtz et al. (2013), que para uma população de $250 \mathrm{mil}$ plantas/ha verificaram a redução do florescimento com o aumento de doses de nitrogênio. As diferenças observadas na bibliografia e entre anos agrícolas no presente experimento se devem, possivelmente, às populações utilizadas e condições meteorológicas. Ao considerar o último aspecto, o menor florescimento observado em 2011 se deve às maiores temperaturas do ar registradas nesse ano agrícola, uma vez que a floração na cultura da cebola está associada principalmente à ocorrência de um somatório de temperaturas baixas (de 9 a $13^{\circ} \mathrm{C}$ ) durante o ciclo de cultivo (Brewster, 2008).

\section{Perda pós-colheita (PPC)}

Em 2011 e 2013, a análise de variância não indicou interação significativa $(p>0,05)$ entre doses de $\mathrm{N}$ e população em relação à perda de peso após quatro meses de armazenamento (PPC).

Em 2011 observou-se que as doses de N não influenciaram a PPC, mas que o aumento da densidade populacional (DS) reduziu linearmente $(\mathrm{p}>0,01)$ a variável $\left(\mathrm{y}_{\mathrm{PCC}, 2011}=29,293265-\right.$ 0,0000264x, $\left.\mathrm{R}^{2}=0,84\right)$. Assim, a PPC passou linearmente de $22,3 \%$ em populações de $300 \mathrm{mil}$ plantas/ha para 13,2\% em DP de 600 mil plantas/ha, em relação ao peso inicial de armazenamento. Possivelmente, tal comportamento possa ser atribuído à formação de bulbos de maior diâmetro transversal e com maior conteúdo de água em menores densidades populacionais. Quanto maior o teor de água de um dado alimento, menor o tempo de conservação, em especial, em condições ambientais não controladas.

Em 2013, os dados da análise de variância não revelaram diferenças significativas $(\mathrm{p}>0,05)$ entre as doses de $\mathrm{N}$ e populações para as perdas de peso após quatro meses de armazenamento. Nesse ano a PPC média foi de 28,8\% em relação ao peso inicial armazenado. Portanto, neste ano agrícola, doses e populações não influenciaram as perdas de peso em pós-colheita.

De acordo com Kurtz et al. (2013), o aumento nas perdas em pós-colheita pelo incremento nas doses de $\mathrm{N}$ tem ocorrido quando há excesso de chuvas. Ao considerar que em 2011 foram obtidas as maiores produtividades e menor PPC sob maior precipitação $(853,5 \mathrm{~mm})$ em relação a 2013 (Figura 1), percebe-se que esta variável meteorológica não influenciou a PPC. Os resultados obtidos são concordantes com os de Hussaini et al. (2000), que não observaram efeitos da adição de $\mathrm{N}$ na conservação dos bulbos pela adição de até $164 \mathrm{~kg} / \mathrm{ha}$.

As variações observadas na PPC entre 2011 e 2013, possivelmente, são devido às variações de temperatura e umidade relativa do ambiente de armazenamento (Honorio \& Moretti, 2002). Contudo, mais estudos são necessários para confirmar essa hipótese, uma vez que no presente estudo as referidas variáveis micrometeorológicas no estaleiro não foram monitoradas durante o período de armazenamento dos bulbos.

Os resultados obtidos permitem concluir que produtividades comerciais totais máximas de 58,3 e 55,1 t/ha são obtidas com doses de 161,3 e 129,0 $\mathrm{kg} / \mathrm{ha}$ de $\mathrm{N}$, respectivamente para 2011 e 2013. O aumento de doses de $\mathrm{N}$ reduz 


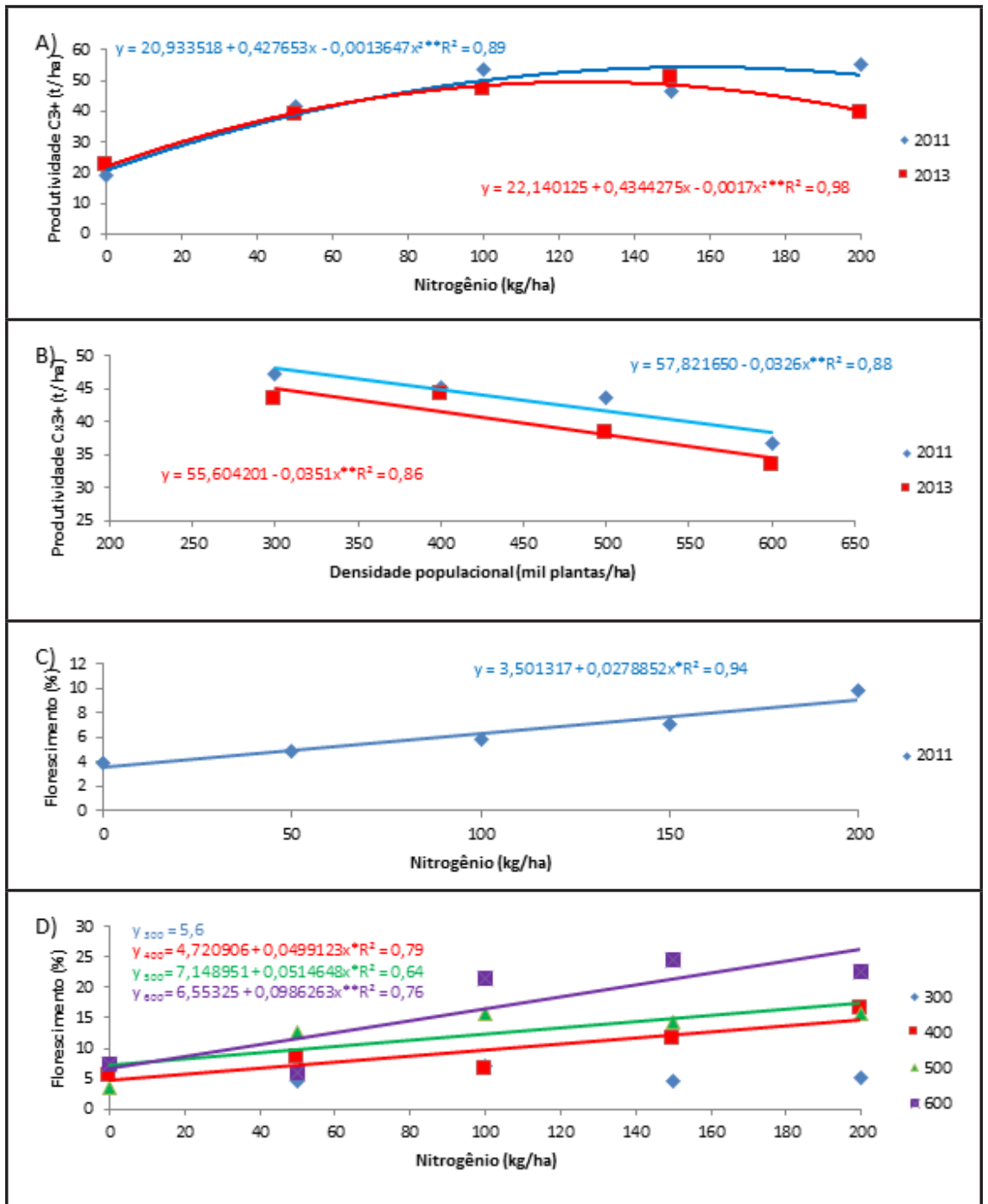

Figura 4. $\mathrm{A}=$ produtividade classe $\mathrm{Cx} 3+\mathrm{x}$ dose de $\mathrm{N}$ em 2011 e 2013 ; $\mathrm{B}=$ classe $\mathrm{Cx} 3+$ $\mathrm{x}$ densidade populacional em 2011 e 2013; $\mathrm{C}=$ percentagem de florescimento $\mathrm{x}$ dose de $\mathrm{N}$ em 2011; $\mathrm{D}=$ percentagem de florescimento $\mathrm{x}$ dose de $\mathrm{N} x$ densidade populacional em $2013(* \mathrm{p}>0,05 ; * * \mathrm{p}>0,01)\{\mathrm{A}=$ yield class $\mathrm{Cx} 3+\mathrm{x} \mathrm{N}$ rate in 2011 and $2013 ; \mathrm{B}=$ class $\mathrm{Cx} 3+\mathrm{x}$ population density in 2011 and 2013; $\mathrm{C}=$ percentage of flowering $\mathrm{x} \mathrm{N}$ rate in 2011; $\mathrm{D}=$ percentage of flowering $\mathrm{x} N$ rate $\mathrm{x}$ population density in $2013(* \mathrm{p}>0,05 ; * * \mathrm{p}>0,01)\}$. Ituporanga, Epagri, 2013.

a formação de bulbos Cx2. A maior produtividade de bulbos Cx3 (25,6 a $39,3 \mathrm{t} / \mathrm{ha}$ ) é obtida com populações de 500 a 600 mil plantas/ha e doses de 126,1 a $135,9 \mathrm{~kg} /$ ha de N. O aumento da densidade populacional reduz a produtividade de bulbos $\mathrm{Cx} 3+$. As maiores produtividades $\mathrm{Cx} 3+(49,6$ a 54,4 t/ha) são alcançadas com doses de 126,3 e $156,7 \mathrm{~kg} / \mathrm{ha}$ de N. Para a cultivar Bola Precoce, teores de $\mathrm{N}$ na faixa de 23,0 a $34,0 \mathrm{~g} / \mathrm{kg}$ podem ser considerados adequados na metade do ciclo. $\mathrm{O}$ aumento das doses de $\mathrm{N}$ concorre para o aumento do florescimento na cultura, mas não influencia as perdas em pós-colheita após quatro meses de armazenamento.

\section{AGRADECIMENTOS}

Os autores agradecem à FAPESC pelo financiamento da pesquisa e auxílio dos colegas da Epagri Técnico Agrícola Marcelo Pitz e Assistentes de Campo Odair Justen e Olindo Andre da Epagri, Estação Experimental de Ituporanga.

\section{REFERÊNCIAS}

ABALOS, D; SANCHEZ-MARTIN, L; GARCIA-
TORRES, L; GROENIGEN, JW; VALLEJO, A. 2014. Management of irrigation frequency and nitrogen fertilization to mitigate GHG and $\mathrm{NO}$ emissions from drip-fertigated crops. Science of Total Environment 490: 880-888.

ABDISSA, Y; TEKALIGN, T; PANT, LM. 2011. Growth, bulb yield and quality of onion (Allium cepa L.) as influenced by nitrogen and phosphorus fertilization on vertisol I. growth attributes biomass production and bulb yield. African Journal of Agricultural Research 6: 3252-3258.

BARBOSA FILHO, MP; COBUCCI, T; FAGERIA, NK; MENDES, PN. 2008. Determinação da necessidade de adubação nitrogenada de cobertura em feijoeiro irrigado com auxílio do clorofilômetro portátil. Ciência Rural 38: 1843-1848.

BRASIL. 1995. Ministério da Agricultura, Abastecimento e Reforma Agrária. Portaria n. 529 de 18 ago. 1995. Diário Oficial da República Federativa do Brasil. Brasília, 1 de set. 1995, Seção 1: 13513.

BREWSTER, JL. 2008. Onions and other vegetables alliums. 2 ed. UK: Wellesbourne. $455 \mathrm{p}$.

CALDWELL, JON; SUMNER, ME; VAVRINA, CS. 1994. Development and testing of preliminary foliar DRIS norms for onions. HortScience 29: 1501-1504.

CANTARELLA, H. 2007. Nitrogênio. In: NOVAIS, RF; ALVAREZ, VH; BARROS, RLFE; CANTARUTTI, RB; NEVES, JCL (eds). Fertilidade do Solo. Viçosa: SBCS, p.375-470.

DELLACECCA, V; LOVATO, AFS. 2000. Effects of different plant densities and planting systems on onion (Allium cepa L.) Bulb quality and yield. Acta Horticulturae 533: 197-204. Disponível em http://www.actahort. org/books/533/533_24.htm. Acessado em 20 de março de 2011.

EMBRAPA. 2006. Sistema brasileiro de classificação de solos. 2. ed. Rio de Janeiro: Embrapa Solos. 306p.

EPAGRI. 2013. Sistema de produção para a cebola - Santa Catarina. Florianópolis: Epagri. 106p. (Sistemas de Produção n.46).

FAYAD, JA; MONDARDO, M. 2004. Determinação das taxas diárias de absorção de nutrientes pela cebola, cv. Crioula, cultivada no sistema de plantio direto de hortaliças. Florianópolis: Epagri. 8p. (Boletim Técnico, 5).

GANDIN, CL; GUIMARÃES, DR; THOMAZELLI, LF. 1994. Caracterização de quatro cultivares de cebola lançadas em Santa Catarina, Brasil. Pesquisa Agropecuária Brasileira 29: 1941-1945.

GANDIN, CL; YOKOYAMA, S; THOMAZELLI, LF; GUIMARÃES, DR; BIASI, J; BECKER, WF; FAORO, ID; NETO, JAZ; PIANA, Z; MÜLLER, JJV; SILVA, ACF; VIZZOTTO, VJ. 1989. Nova cultivar de cebola de ciclo médio para SC. Agropecuária Catarinense 2: 40-42.

GATTO, RF. 2013. Produtividade da cultura da cebola sob doses de nitrogênio e lâminas de 
irrigação por gotejamento. Lavras: UFLA. 82p (Dissertação mestrado).

GONÇALVES, PAS; MISSIO, VC; KURTZ, C; VIEIRA NETO, J. 2014. Relação dos nutrientes foliares com a incidência de trips nos cultivares de cebola Epagri 352-Bola Precoce e Epagri 362-Crioula Alto Vale. Agropecuária Catarinense 26: 86-90.

HONORIO, SL; MORETTI, CL. 2002. Fisiologia pós-colheita de frutas e hortaliças. In: CORTEZ, LAB, HONÓRIO, SL; MORETTI, CL (eds). Resfriamento de frutas e hortaliças. Brasília: Embrapa Informação Tecnológica/ Embrapa Hortaliças. p. 59-81.

HUSSAINI, MA; AMANS, EB; RAMALAN, AA. 2000. Yield, bulb size distribution and storability of onion (Allium cepa $\mathrm{L}$.) under different levels of $\mathrm{N}$ fertilization and irrigation regime. Tropical Agriculture 77:145-149.

JONES JUNIOR, JB; WOLF, B; MILLS, HA. 1991. Plant analysis and analysis handbook: a practical sampling preparation, analysis and interpretation guide. Athen: MicroMacro. 213p.

KHAN, H; IQBAL, M; GHAFFOOR, A; WASEEM, K. 2002. Effect of various plant spacing and different nitrogen levels on the growth and yield of onion (Allium cepa L.). Journal of Biological Sciences 2: 545-547.

KURTZ, C; ERNANI, PR; PAULETTI, V; MENEZES JUNIOR, FOG; VIEIRA NETO, J. 2013. Produtividade e conservação de cebola afetadas pela adubação nitrogenada no sistema de plantio direto. Horticultura Brasileira 31: 559-567.
MALAVOLTA, E; VITTI, GC; OLIVEIRA, SA. 1997. Avaliação do estado nutricional das plantas: princípios e aplicações. 2 ed. Piracicaba: POTAFOS. 319p.

MANSOURI, H; BANNAYAN, M; MOGHADDAM, PR; LAKZIAN, A. 2014. Management of nitrogen fertilizer, irrigation and plant density in onion production using response surface methodology as optimization approach. African Journal of Agricultural Research 9: 676-687.

MARSCHNER, H. 2012. Mineral nutrition of higher plants. 3 ed. London: Academic Press. $651 \mathrm{p}$.

MAY, A; CECÍLIO FILHO, AB; PORTO, DRQ; VARGAS, PF; BARBOSA, JC. 2007. Produtividade de híbridos de cebola em função da população de plantas e da fertilização nitrogenada e potássica. Horticultura Brasileira 25: 053-059.

MENEZES JÚNIOR, FOG; VIEIRA NETO, J. 2012. Produção da cebola em função da densidade de plantas. Horticultura Brasileira 30: 733-739.

RESENDE, GM; COSTA, ND. 2009. Produtividade e armazenamento de cebola (Allium cepa L.) submetida a doses de nitrogênio e potássio via fertirrigação em cultivo de verão. Ciência e Agrotecnologia 33: 1314-1320.

RESENDE, GM; COSTA, ND; PINTO, JM. 2009. Rendimento e conservação pós- colheita de bulbos de cebola com doses de nitrogênio e potássio. Horticultura Brasileira 27: 139-143. RUSSO, VM. 2008. Plant density and nitrogen fertilizer rate on yield and nutrient content of onion developed from greenhouse-grown transplants. HortScience 43: 1759-1764.

SHOCK, CC; FEIBERT, EBG; SAUNDERS, L. 2004. Plant population and nitrogen fertilization for subsurface drip-irrigated onion. HortScience 39: 1722-1727.

SHOJAEI, H; VAKILI, SMA; KHODADADI, M; MIRZAEI, Y. 2011. Effect of different N fertilization levels and plant-population on agronomic traits and decrease in bolting of autumn-sown onion in Shahdad Region of Kerman, Iran. Plant Ecophysiology 3: 59-64.

STOFFELLA, PJ. 1996. Planting arrangement and density of transplants influence sweet Spanish onion yields and bulbs size. Hortscience 31 : 1129-1130.

TEDESCO, MJ; GIANELLO, C; BISSANI, CA; BOHNEN, H. 1995. Análise de solo, plantas e outros materiais. 2.ed. Porto Alegre: UFRGS. 174p. (Boletim Técnico, 5).

VILAS BOAS, RC; CARVALHO, JG; PEREIRA, GM; SOUZA, RJ; GAMA, GBN; GARCIA, HH; ARAUJO, RAS. 2014. Rendimento da cultura da cebola submetida a níveis de água e nitrogênio por gotejamento. Semina 35: 633-646.

WANG, Z; LI, J; LI, Y. 2014. Effects of drip irrigation system uniformity and nitrogen applied on deep percolation and nitrate leaching during growing seasons of spring maize in semi-humid region. Irrigation Science 32: 221-236.

ZONTA, EP; MACHADO, AA. 1984. SANEST - Sistema de Análise Estatísticas para Microcomputadores. Pelotas: UFPel. 75 p. 